Research Article

\title{
CRLH Transmission Lines for Telecommunications: Fast and Effective Modeling
}

\author{
Juanjuan Gao and Guizhen Lu \\ School of Information Engineering, Communication University of China, Beijing 100024, China \\ Correspondence should be addressed to Juanjuan Gao; gaojj_1989@126.com
}

Received 19 August 2016; Revised 21 November 2016; Accepted 15 December 2016; Published 4 January 2017

Academic Editor: Davide Ramaccia

Copyright (C) 2017 J. Gao and G. Lu. This is an open access article distributed under the Creative Commons Attribution License, which permits unrestricted use, distribution, and reproduction in any medium, provided the original work is properly cited.

\begin{abstract}
A different parameter extraction approach based on zero immittances for composite right/left-handed (CRLH) structure is presented. For lossless unit cell equivalent circuit model, LC parameters of series and parallel branches are extracted according to series resonance frequency and parallel resonance frequency, respectively. This approach can be applied to symmetric and unbalanced CRLH structures. The parameter extraction procedure is provided and validated by T-type unit cell model. The responses of distributed prototype and extracted equivalent LC circuit model are in good agreement. The equivalent circuit modeling can improve the degree of freedom in the CRLH TLs design. This parameter extraction method provides an effective and straightforward way in CRLH metamaterials design and applications in telecommunication systems.
\end{abstract}

\section{Introduction}

In telecommunication system, the role of the oscillator is to establish stable harmonic oscillation at a specific carrier frequency for modulation and mixing, which is shown in Figure 1(a). In the oscillator circuit, the main function of resonator is frequency selection and storage of energy. Conventional transmission line resonator is size dependent on frequency, which is not suitable for use in miniaturized integrated circuits. Composite right/left-handed transmission line (CRLH TL), introduced by Caloz and Itoh, is the transmission line (TL) approach of left-handed materials (LHMs) [1]. CRLH metamaterials with right-handed (RH) and left-handed (LH) properties have the advantages of low loss and wide bandwidth, which have been extensively studied and broad applied in guided-wave and radiatedwave devices for over decades [2-5]. Typically, the distributed CRLH TL is consisting of the series interdigital capacitor (IDC) and the shunt shorted stub inductor (SSI), which can be realized by the microstrip line and coplanar waveguide [4]. Based on CRLH TL structures zeroth-order resonator can be achieved; its size is frequency independent and can be realized, theoretically, arbitrarily small [6]. The schematic diagram of oscillator circuit using a zeroth-order resonator is shown in Figure 1(b). Using the zeroth-order resonator can effectively reduce the size of the oscillator circuit and improve the miniaturization of communication systems.

To accurately design CRLH TL and investigate the characteristics of CRLH TL-based devices, the equivalent circuit model is used to parameterize the complicated behaviors of the practical distributed circuits $[7,8]$. The characteristics of the CRLH TLs are closely related to the LC parameters. Accurate design depends on whether the parameters meet the requirements. The design can be modified by parameter extraction in order to achieve optimum performances. In [9], to calculate the extracted LC parameters, the equivalent $\mathrm{T}$ and $\Pi$ networks of IDC and SSI are separately characterized by admittance and impedance matrices; however coupling effects between IDC and SSI are not taken into account in this parameter extraction procedure. Otto et al. proposed an energy based method to extract equivalent circuit parameters [10], which was further implemented in [11]. However, this method requires the use of software for electromagnetic energy calculation, cannot be extracted by experiment. Based on effective medium concept, revised Nicolson-Ross-Weir (NRW) approach was applied to extract equivalent parameters. In this method, the equivalent circuit model parameters are deduced by the effective permittivity $\varepsilon_{r}$ and the effective 


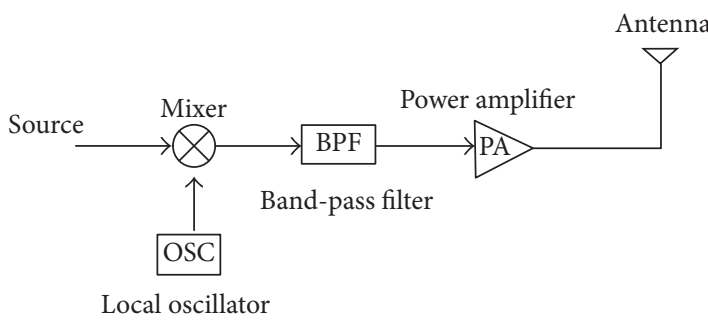

(a)

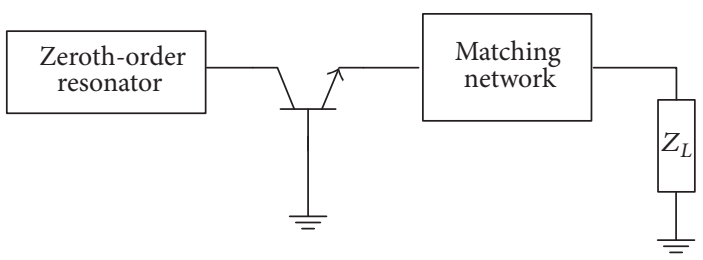

(b)

Figure 1: (a) The block diagram of telecommunication system. (b) The block diagram of oscillator.

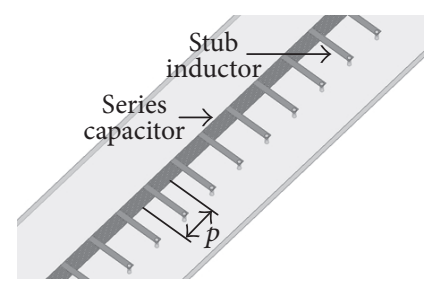

(a)

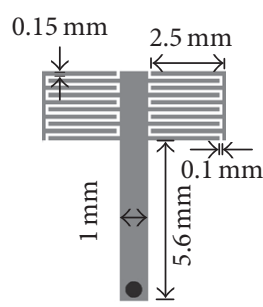

(b)

FIgURE 2: CRLH TL structures. (a) Periodic CRLH TLs with series capacitors and shunt stub inductors. (b) Geometry of unit cell of CRLH TL.

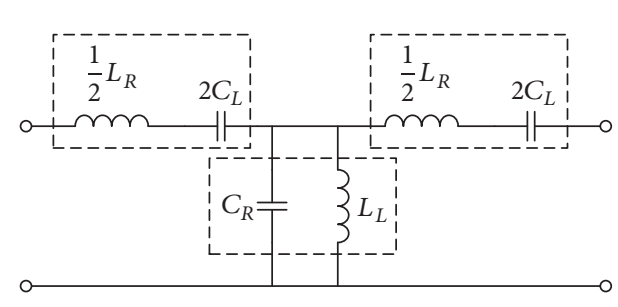

(a)

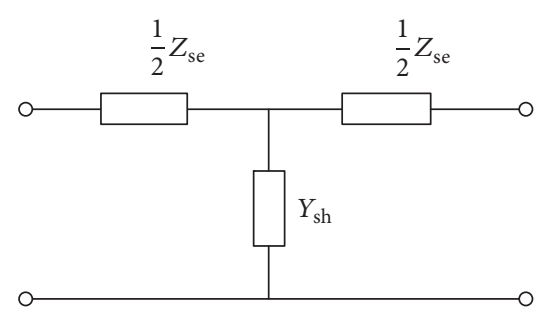

(b)

FIgURE 3: Equivalent circuit models of CRLH TL for parameters extraction. (a) T-type unit cell circuit model. (b) Equivalent network.

permeability $\mu_{r}$. However, this method suffers multisolutions problem [12-15]. The equivalent circuit extraction method proposed in [16] employs the transmission-line-reflect (TLR) calibration to deembed the effects of the transmission lines. In this method both full-wave simulation and measured $S$ parameters are used to obtain the $A B C D$ transmission matrix of the CRLH unit cell.

In this paper, a different parameter extraction approach based on zero immittances has been presented. For lossless equivalent LC circuit model adopted, series resonance frequency and parallel resonance frequency are obtained from immittances characteristic, which is utilized to extract the LC parameters. In the proposed method, test transmission lines are placed in both sides of the CRLH structure. The effects of the transmission lines are eliminated by matrix calculation. The scattering parameter used can be obtained by full-wave simulations. The extraction procedure is convenient and effective and can be applied in unbalanced CRLH structures. This approach is illustrated by T-type unit cell model. The transmission characteristics of the distributed CRLH structure and the extraction LC equivalent circuit are in comparison with each other, and in wider bandwidth the extracted equivalent LC circuit model is effective.

\section{Equivalent Circuit Modeling and Parameter Extraction Approach}

The CRLH TL considered in this paper is constituted by series interdigital capacitor and parallel shorted stub inductors, which is shown in Figure 2(a). The dimensions are the same as those in [1], which is depicted in Figure 2(b). Assuming the transmission line is lossless, interdigital capacitor is modeled with $C_{L}$ series $L_{R}$ and stub inductor is modeled with $L_{C}$ shunt $C_{R}$. The T-type unit cell circuit model of CRLH TLs is shown in Figure 3(a) and the corresponding microwave network is displayed in Figure 3(b).

Due to the unit cell circuit model shown in Figure 3, the immittances $Z_{\mathrm{se}}$ and $Y_{\mathrm{sh}}$ characteristics are plotted in 


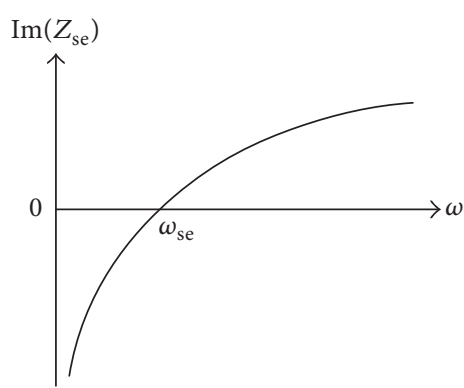

(a)

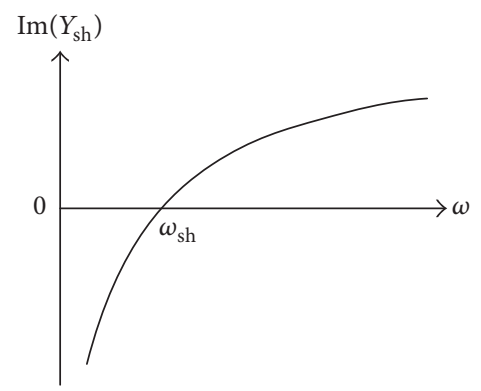

(b)

FIGURE 4: Immittances characteristics of T-type equivalent circuit model. (a) Series impedance $Z_{\text {se }}$ characteristic curve. (b) Shunt admittance $Y_{\text {sh }}$ characteristic curve.

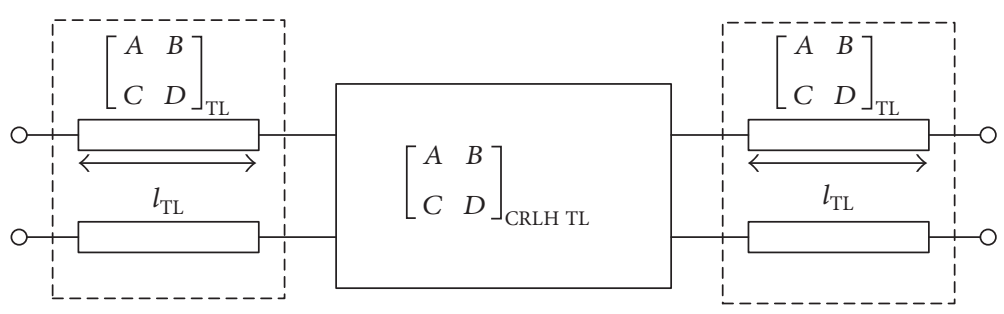

FIGURE 5: Parameter extraction circuit system model.

Figure 4 . When we have zero immittances, the series resonance frequency $\omega_{\text {se }}$ and parallel resonance frequency $\omega_{\text {sh }}$ are obtained.

$$
\begin{aligned}
& \omega_{\mathrm{se}}=\left.\omega\right|_{\operatorname{Im}\left(Z_{\mathrm{se}}\right)=0}=\frac{1}{\sqrt{L_{R} C_{L}}}, \\
& \omega_{\mathrm{sh}}=\left.\omega\right|_{\operatorname{Im}\left(Y_{\mathrm{sh}}\right)=0}=\frac{1}{\sqrt{L_{C} C_{R}}} .
\end{aligned}
$$

Based on the characteristics of immittances $Z_{\text {se }}$ and $Y_{\text {sh }}$, we introduce an extraction approach to yield simple expressions for $C_{L}, L_{R}, L_{C}$, and $C_{R}$. For a two-port network, depicted in Figure 2(b), $Z_{\text {se }}$ and $Y_{\text {sh }}$ can be described in terms of $A B C D$ transmission matrix. Using standard network conversion formulas, the series impedance and parallel admittance can be written in terms of the $A B C D$ parameters, which lead to

$$
\begin{aligned}
& Z_{\mathrm{se}}^{\text {sym }}=\frac{2(A-1)}{C}, \\
& Y_{\mathrm{sh}}^{\text {sym }}=C .
\end{aligned}
$$

With zero immittances $Z_{\text {se }}$ and $Y_{\text {sh }}$, the series resonance frequency and the parallel resonance frequency are determined by

$$
\begin{aligned}
& \left.\operatorname{Im}\left(Z_{\mathrm{se}}\right)\right|_{\omega_{\mathrm{se}}}=0, \\
& \left.\operatorname{Im}\left(Y_{\mathrm{sh}}\right)\right|_{\omega_{\mathrm{sh}}}=0 .
\end{aligned}
$$

With $\omega_{\mathrm{se}}$ and $\omega_{\mathrm{sh}}$, the LC parameters of series and parallel tank would be solved separately. And the corresponding extract parameters are determined by

$$
\begin{aligned}
L_{R} & =\left.\frac{1}{2} \frac{\partial}{\partial \omega} \operatorname{Im}\left(Z_{\mathrm{se}}\right)\right|_{\omega_{\mathrm{se}}}, \\
C_{L} & =\frac{1}{\omega_{\mathrm{se}}^{2} L_{\mathrm{se}}}, \\
C_{R} & =\left.\frac{1}{2} \frac{\partial}{\partial \omega} \operatorname{Im}\left(Y_{\mathrm{sh}}\right)\right|_{\omega_{\mathrm{sh}}}, \\
L_{L} & =\frac{1}{\omega_{\mathrm{sh}}^{2} L_{\mathrm{sh}}} .
\end{aligned}
$$

When $\omega_{\text {se }}=\omega_{\text {sh }}$, called balanced case, CRLH TLs would exhibit special properties. In practice, most initial fabrics are unbalanced, and with this extraction approach we can conveniently revise the design. That is, this extraction procedure is applicable for both balanced and unbalanced cases.

\section{Extraction Procedure and Validation}

This section provides the extraction procedure based on circuit model shown in Figure 3 and verifies this method with CRLH TLs proposed by Caloz and Itoh. Usually, in order to obtain the transmission matrix of CRLH TL, a section of microstrip transmission line (TL) has to be added at each end of the component in full-wave simulation; the diagram is shown in Figure 5. However, the added microstrip TLs would change the original network characteristics such as 


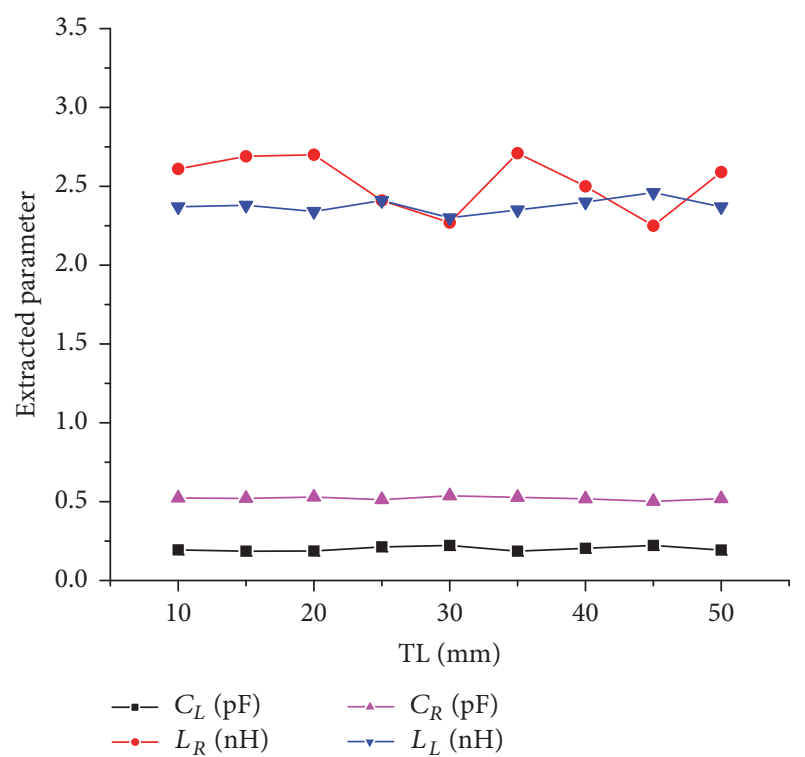

FIGURE 6: Extracted LC parameters of equivalent LC circuit model.

propagation phase. Therefore it is essential to eliminate the effect due to the test TLs.

The distributed CRLH TL and extracted equivalent circuit model are validated by comparing the scattering characteristics. The procedure is provided as follows:

(1) Do full-wave simulation for the distributed circuit of Figure 5 and test microstrip TL to get the $S$ parameters of overall circuit and TLs, respectively.

(2) Compute $[A B C D]$ of overall circuit and $[A B C D]$ of TL corresponding to the $S$-parameters obtained in (1) using standard network conversion formulas.

(3) Compute $[A B C D]$ of CRLH TL using

$$
\begin{aligned}
& {[A B C D]_{\text {overall }}} \\
& \quad=[A B C D]_{\mathrm{TL}}[A B C D]_{\mathrm{CRLH} T L}[A B C D]_{\mathrm{TL}} .
\end{aligned}
$$

(4) Compute the $Z_{\text {se }}$ and $Y_{\text {sh }}$ vector using again standard network conversion formulas.

(5) Find zero points of $Z_{\mathrm{se}}$ and $Y_{\mathrm{sh}}$ using interpolation method to determine series resonance frequency $\omega_{\text {se }}$ and parallel resonance frequency $\omega_{\text {sh }}$.

(6) Compute the $C_{L}, L_{R}, L_{C}$, and $C_{R}$ using (4).

(7) With extraction parameters obtained from (6), simulate the equivalent LC circuit model and compare with distributed CRLH TL.

The CRLH TLs proposed by Caloz and Itoh and shown in Figure 1 is considered here to illustrate the parameter extraction procedure with T-type unit cell circuit model. Fullwave simulation and numerical calculation are developed; the extraction results are presented in Figure 6. With different test TL lengths, the extracted LC parameters are mainly stable. It should be noted that, since the test transmission

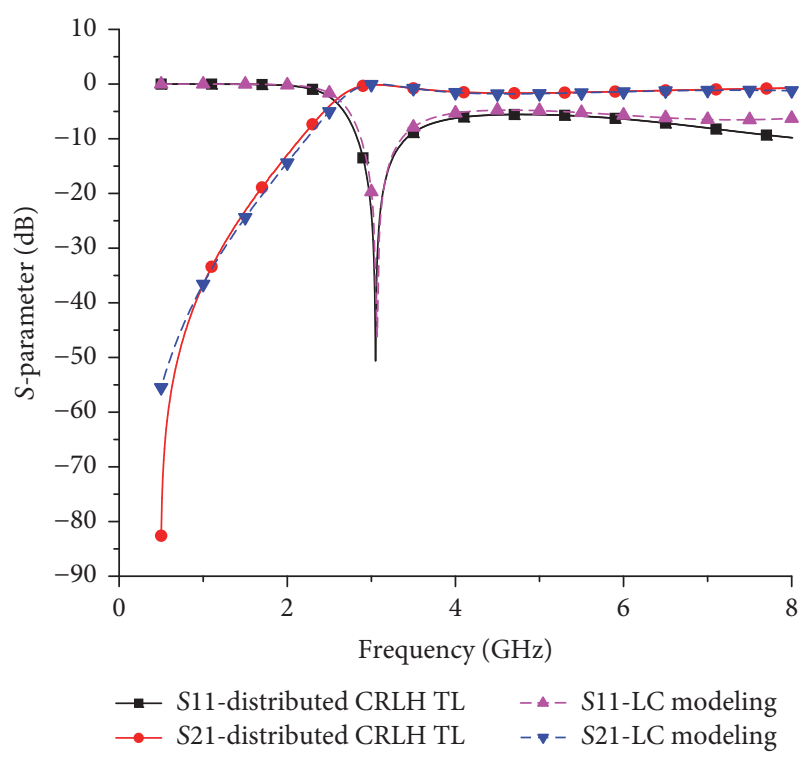

FIgURE 7: Comparison of $S$-parameters between distributed 1-cell CRLH TL and equivalent LC circuit model.

lines are series with the interdigital capacitor, the different transmission line length has a great influence on the $L_{R}$ parameter. In the process of parameter extraction, the $L_{R}$ value can be obtained by multiple extractions and averaging.

The set parameters $L_{R}=2.56 \mathrm{nH}, C_{L}=0.192 \mathrm{pF}, C_{R}=$ $0.523 \mathrm{pF}$, and $L_{L}=2.33 \mathrm{nH}$ of T-type unit cell circuit with $\mathrm{TL}=20 \mathrm{~mm}$ has been selected for comparison with practical distributed structure. In Figure 7, S-parameters of distributed 1-cell CRLH TL structure and equivalent LC circuit model are presented. As shown in comparison results, within wider bandwidth, they are in good agreement with each other.

The CRLH TL is balanced when the series and shunt resonant frequencies are equal; on the contrary, the CRLH TL is unbalanced when the series and shunt resonant frequencies are different. It is noted that the series and parallel LCs are extracted from $\omega_{\text {se }}$ and $\omega_{\text {sh }}$, respectively. Regardless of whether the series resonant frequency and the parallel resonant frequency are equal, this extraction method can model the CRLH TLs. This makes it convenient to apply this approach in both balanced and unbalanced CRLH structures.

\section{Conclusion}

A zero immittances based parameter extraction approach for distributed CRLH structures has been proposed, which is applicable to symmetric unit cells. According to series resonance frequency $\omega_{\text {se }}$ and parallel resonance frequency $\omega_{\text {sh }}$, determined by immittances characteristics, LCs of series branch and parallel branch are extracted, respectively, and therefore this method can be used in unbalanced CRLH structures. The presented extraction procedure provides an effective and straightforward way in CRLH metamaterials design and application of telecommunication system. Finally, the authors are currently applying this approach in analysis of CRLH metamaterials-based microwave circuit. 


\section{Competing Interests}

The authors declare that there are no competing interests regarding the publication of this paper.

\section{References}

[1] C. Caloz and T. Itoh, "Novel microwave devices and structures based on the transmission line approach of metamaterials," in Proceedings of the IEEE MTT-S International Microwave Symposium Digest, vol. 1, pp. 195-198, Philadelphia, Pa, USA, June 2003.

[2] A. Lai, C. Caloz, and T. Itoh, "Composite right/left-handed transmission line metamaterials," IEEE Microwave Magazine, vol. 5, no. 3, pp. 34-50, 2004.

[3] J. Sorocki, I. Piekarz, K. Wincza, and S. Gruszczynski, "Right/left-handed transmission lines based on coupled transmission line sections and their application towards bandpass filters," IEEE Transactions on Microwave Theory and Techniques, vol. 63, no. 2, pp. 384-396, 2015.

[4] C. Caloz, A. Sanada, and T. Itoh, "A novel composite right/left-handed coupled-line directional coupler with arbitrary coupling level and broad bandwidth," IEEE Transactions on Microwave Theory and Techniques, vol. 52, no. 3, pp. 980-992, 2004.

[5] J.-G. Lee and J.-H. Lee, "Zeroth order resonance loop antenna," IEEE Transactions on Antennas and Propagation, vol. 55, no. 3, pp. 994-997, 2007.

[6] A. Sanada, C. Caloz, and T. Itoh, "Zeroth-order resonance in composite right/left handed transmission line resonators," in Proceedings of the Asia-Pacific Microwave Conference (APMC '03), pp. 1588-1592, Seoul, Republic of Korea, 2003.

[7] F. Aznar, M. Gil, J. Bonache et al., "Characterization of miniaturized metamaterial resonators coupled to planar transmission lines through parameter extraction," Journal of Applied Physics, vol. 104, no. 11, Article ID 114501, 2008.

[8] S.-G. Mao, S.-L. Chen, and C.-W. Huang, "Effective electromagnetic parameters of novel distributed left-handed microstrip lines," IEEE Transactions on Microwave Theory and Techniques, vol. 53, no. 4, pp. 1515-1521, 2005.

[9] C. Caloz and T. Itoh, "Transmission line approach of lefthanded (LH) materials and microstrip implementation of an artificial LH transmission line," IEEE Transactions on Antennas and Propagation, vol. 52, no. 5, pp. 1159-1166, 2004.

[10] S. Otto, A. Rennings, T. Liebig, C. Caloz, and K. Solbach, "An energy-based circuit parameter extraction method for CRLH leaky wave antennas," in Proceedings of the 4th European Conference on Antennas and Propagation (EuCAP '10), pp. 1-5, Barcelona, Spain, April 2010.

[11] S. Otto, A. Rennings, K. Solbach, and C. Caloz, "Transmission line modeling and asymptotic formulas for periodic leaky-wave antennas scanning through broadside," IEEE Transactions on Antennas and Propagation, vol. 59, no. 10, pp. 3695-3709, 2011.

[12] S.-G. Mao, M.-S. Wu, Y.-Z. Chueh, and C. H. Chen, "Modeling of symmetric composite right/left-handed coplanar waveguides with applications to compact bandpass filters," IEEE Transactions on Microwave Theory and Techniques, vol. 53, no. 11, pp. 3460-3466, 2005.

[13] P. Chen, J. Ding, and X. Zheng, "Modeling of asymmetric and unbalanced composite right/left-handed transmission line," in 2011 IEEE International Conference on Signal Processing,
Communications and Computing, ICSPCC 2011, chn, September 2011.

[14] V. Milosevic, B. Jokanovic, and R. Bojanic, "Effective electromagnetic parameters of metamaterial transmission line loaded with asymmetric unit cells," IEEE Transactions on Microwave Theory and Techniques, vol. 61, no. 8, pp. 2761-2772, 2013.

[15] F. J. Hsieh and W. C. Wang, "Full extraction methods to retrieve effective refractive index and parameters of a bianisotropic metamaterial based on material dispersion models," Journal of Applied Physics, vol. 112, no. 6, 2012.

[16] T. Liebig, A. Rennings, S. Held, and D. Erni, "Accurate parameter extraction of lossy composite right/left-handed (CRLH) transmission lines for planar antenna applications," in Proceedings of the 4th International Congress on Advanced Electromagnetic Materials in Microwaves and Optics, Metamaterials, September 2010. 


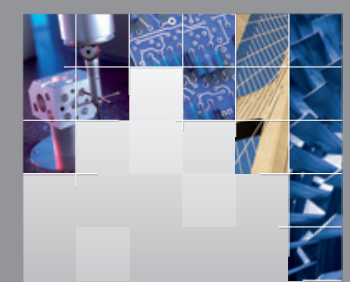

\section{Enfincering}
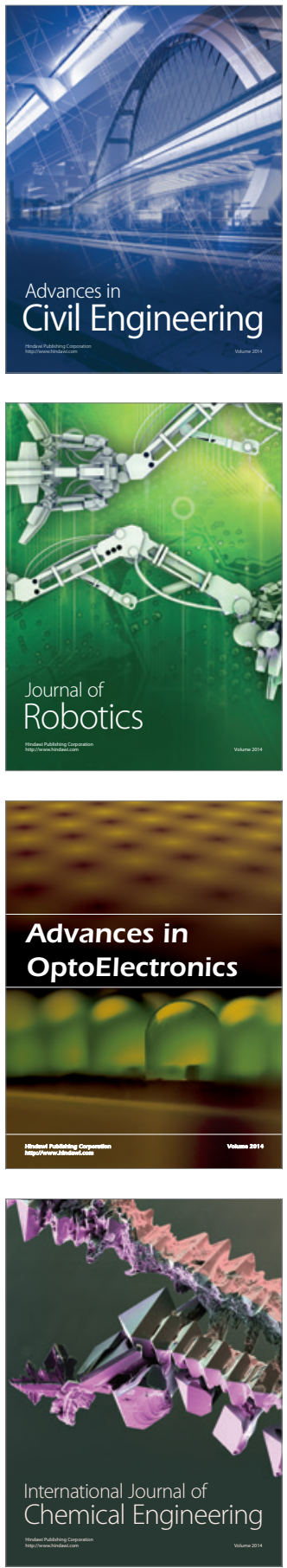

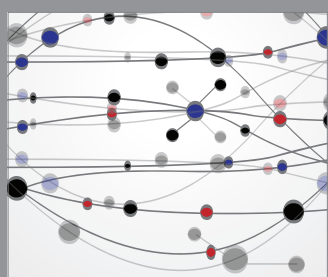

The Scientific World Journal

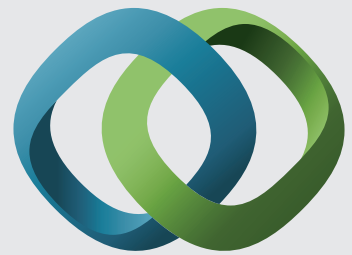

\section{Hindawi}

Submit your manuscripts at

https://www.hindawi.com
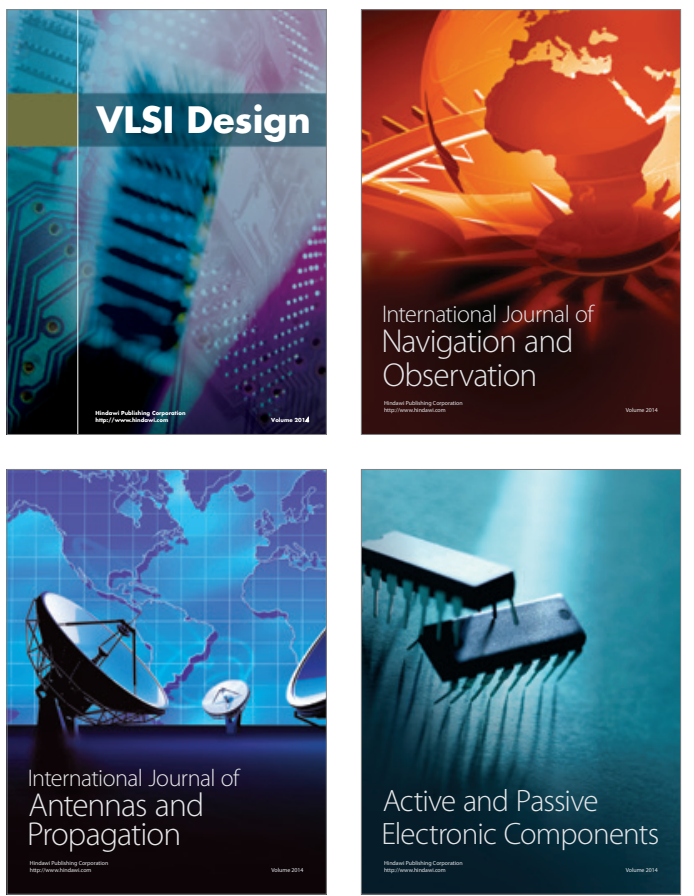
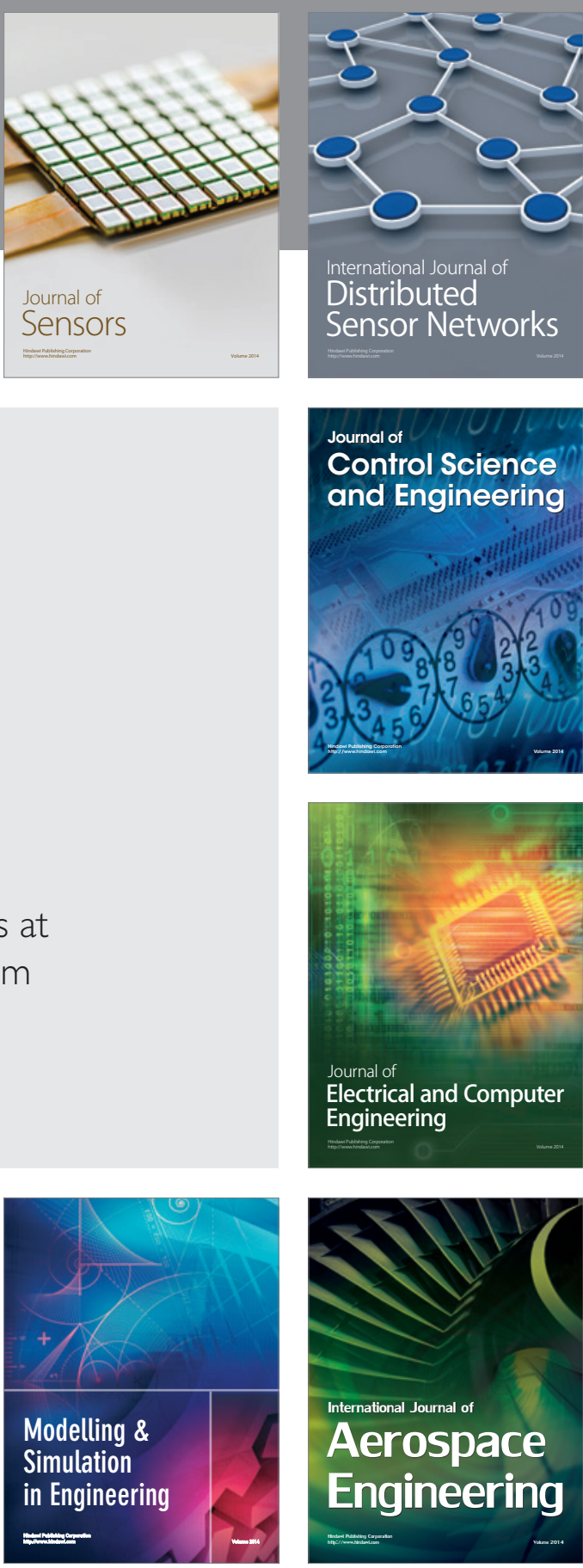

International Journal of

Distributed

Sensor Networks

$-$

Joumal of

Control Science

and Engineering
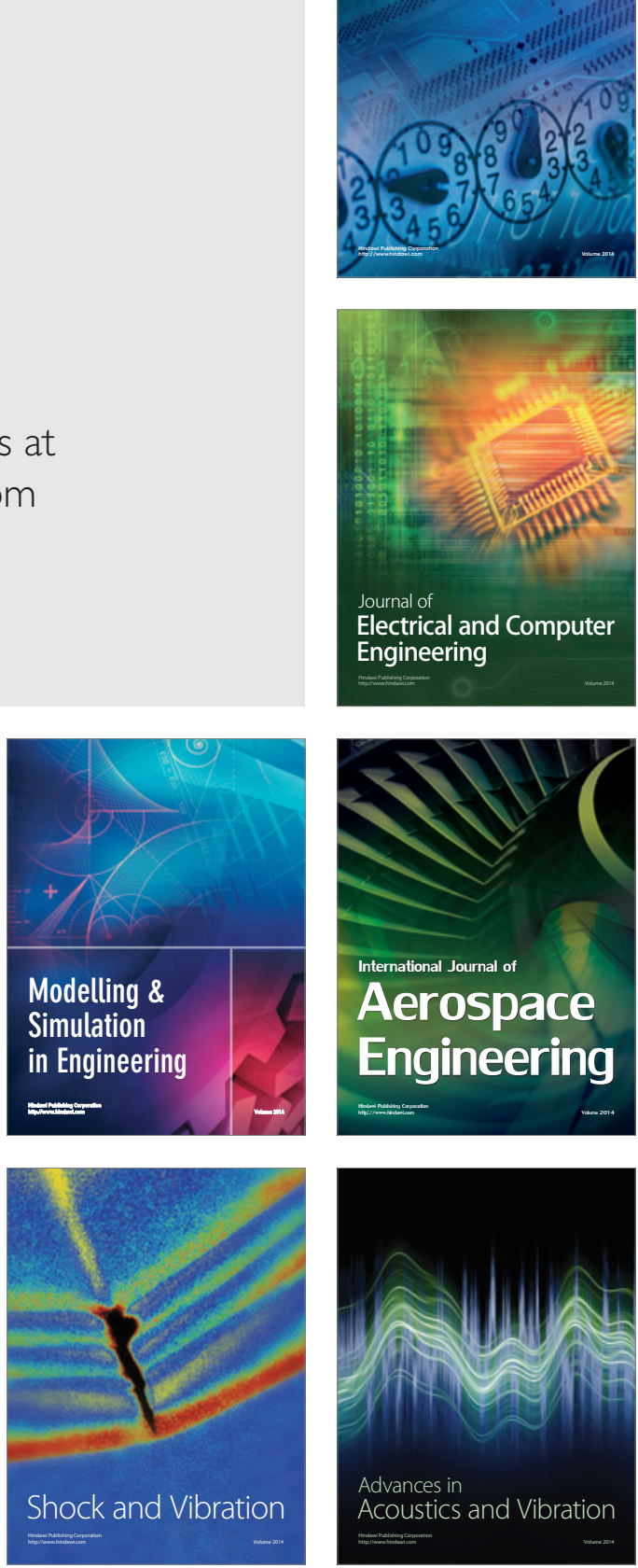